\title{
Using Chinese Remainder Theorem for the Grouping of RFID Tags
}

\author{
Yi-Sheng Su and Ozan K. Tonguz
}

\begin{abstract}
In this paper, we propose a novel scheme for the design of grouping of radio-frequency identification (RFID) tags, based on the Chinese remainder theorem (CRT). Grouping allows verifying the integrity of a collection of objects without the requirement for accessing external systems, and can be extended to identify missing objects. Motivated by the redundancy property of the Chinese remainder representation, we propose grouping of RFID tags by the CRT. The proposed scheme not only provides designated decoding guarantees, but also offers flexibility in constructing group generation matrices. We also characterize the key objects needed to study decoding guarantees of grouping and their extended counterpart, called rank-deficient and deadend sets, respectively, which enable theoretical analyses of error rates. The two key objects are related to the minimum and stopping distances of a linear code, respectively. As such, the characterization offers direct connection with coding theory that helps with the understanding of the verification/identification problems being studied. Theoretical and simulation results are presented, demonstrating that the proposed scheme is an efficient approach to the design of grouping of RFID tags.
\end{abstract}

Index Terms-Grouping of radio-frequency identification (RFID) tags, integrity, missing-object identification, Chinese remainder theorem (CRT).

\section{INTRODUCTION}

Radio-frequency identification (RFID) [1], [2] is the use of any device that can be detected wirelessly using radiofrequency technology. An RFID system typically consists of several tags and one interrogator. Tags are attached to physical objects and are the components that store information about the physical objects, while the interrogator is used to read information from tags wirelessly.

RFID technology has been used in many applications. Among those applications such as incoming shipment inspection, verifying the integrity of a collection of RFID tags or identifying missing RFID tags is beneficial to locate missing RFID tagged objects. In existing RFID systems, these functionalities are realized by looking up the shipment list in Electronic Data Interchange (EDI) [3]. There are other ways to accomplish verification/identification of a collection of RFID tags. In [4], Juels studied the integrity of a pair of RFID tags from the security perspective and introduced the concept of a yoking-proof by exchanging a message authentication code between RFID tags and an external verifier. The yoking-proof was improved and generalized for a collection of RFID tags

This work has been supported by the National Science Council (NSC), Taiwan, ROC, under Contract NSC-102-2221-E-309-003.

Y.-S. Su is with the Department of Computer Science and Information Engineering, Chang Jung Christian University, Tainan, Taiwan, ROC.

O. K. Tonguz is with the Department of Electrical and Computer Engineering, Carnegie Mellon University, Pittsburgh, PA 15213-3890 USA. in [5] and [6]. The work in [7] studied the reliability issues of RFID systems and proposed a method for maintaining systematic reliability based on reading results from multiple interrogators and real-world constraints stored in a database. In [8], Potdar et al. proposed a scheme to address the issue of whether or not all the RFID tags are read based on reading results and gross weights of grouped objects stored in a database. In all of these verification/identification approaches, the access via a network connection to an external system, verifier or database is required.

To eliminate the need for accessing external systems, Sato et al. [9], [10] proposed a new verification scheme for a collection of RFID tags, also known as grouping ${ }^{1}$ of RFID tags, or grouping for short, which can determine the number of missing RFID tags. They also extended grouping to identify the unique identifiers (IDs) of missing RFID tags [11]. The fundamental idea behind grouping and its extended counterpart is to use group generation matrices to divide a target collection of RFID tags into overlapping subcollections, or groups, such that the number of missing RFID tags can be determined or missing RFID tags can be identified. In general, it is desirable to have either guaranteed determination of the number of missing RFID tags or guaranteed identification of missing RFID tags. For these purposes, group generation matrices used in grouping and its extended counterpart need to have certain properties. Grouping and its extended counterpart in [9]-[11], however, did not address such an issue and just used the parity-check matrices of Gallager's low-density paritycheck (LDPC) codes as group generation matrices, henceforth referred to as the LDPC-based scheme. Due to the pseudorandom nature of Gallager's LDPC codes, the LDPC-based scheme cannot easily achieve designated decoding guarantees. To overcome the shortcomings of the LDPC-based scheme, Su et al. [12] recently proposed a scheme based on strongly selective families (SSFs), called the SSF-based scheme, to construct group generation matrices. Although the SSF-based scheme can provide designated decoding guarantees more easily than the LDPC-based one, there is room for improvement. In addition, the work in [12] has not yet fully disclosed the characterization of decoding guarantees of grouping and its extended counterpart.

In this paper, we propose a novel scheme for the design of grouping, based on the Chinese remainder theorem (CRT). By the CRT, the residues of a nonnegative integer modulo pairwise relatively prime integers can form a redundant representation

\footnotetext{
${ }^{1}$ To avoid confusion with coding techniques with algebraic groups, grouping is used instead of group coding [9]-[11].
} 
of the nonnegative integer. We utilize such a redundancy property to construct group generation matrices. The proposed scheme not only provides designated decoding guarantees, but also offers flexibility in constructing group generation matrices. We also characterize the key objects needed to study decoding guarantees of grouping and its extended counterpart, called rank-deficient and dead-end sets, respectively, which enable theoretical analyses of error rates. The two key objects are related to the minimum and stopping distances of a linear code, respectively. As such, the characterization offers direct connection with coding theory that helps in the understanding of the verification/identification problems being studied. Theoretical and simulation results are presented, demonstrating that the proposed scheme is an efficient approach to the design of grouping.

The remainder of this paper is organized as follows. Section II provides a brief overview of grouping and its extended counterpart. The proposed scheme of this paper is presented in Section III, while the key objects needed to study decoding guarantees as well as theoretical analyses of error rates are presented in Section IV. Theoretical and simulation results are given in Section V. Finally, conclusions are drawn in Section VI.

Notations: In this paper, matrices are written in bold uppercase letters, while arrays/vectors are denoted by bold lowercase letters. The columns of a matrix $\mathbf{A}$ are numbered from left to right with the first column index being one and the $i^{\text {th }}$ column, or column $i$, of $\mathbf{A}$ is denoted by $\mathbf{a}_{i}$. Similarly, the rows of a matrix are numbered from top to bottom with the first row index being one. $\mathbf{0}$ is the zero matrix/vector. For any two binary vectors $\mathbf{x}$ and $\mathbf{y}$, we write $\mathbf{x} \oplus \mathbf{y}$ to denote their componentwise exclusive or (XOR). $[n]$ denotes the set $\{1, \ldots, n\} .\lfloor x\rfloor$ is the largest integer not greater than $x \cdot \operatorname{rank}(\mathbf{A})$ is the rank of a matrix $\mathbf{A}$. The cardinality of a finite set $\mathbb{M}$ is denoted as $|\mathbb{M}|$.

\section{Preliminaries}

In this section, we briefly introduce grouping and its extended counterpart. For comparison with [9]-[12] and to keep the paper self-contained, we also give a short introduction to both the LDPC- and SSF-based schemes. For details, refer to [9]-[12].

\section{A. Grouping}

Grouping employs the check sum technique to check the integrity of a group of RFID tags. The check sum of a group of RFID tags is calculated as the componentwise XOR of all hashes of (the unique IDs of) the RFID tags belonging to the group, where a hash function (HF) is used to avoid zero check sums, and it serves as an ID of the group, i.e., group ID. In this context, the hashes of RFID tags and group IDs are binary strings, i.e., strings of 0's and 1's, and, thus, can be viewed as binary row vectors.

1) Encoding: To determine the number of RFID tags missing from a target collection of RFID tags, grouping divides the target collection into overlapping subcollections, i.e., overlapping groups of RFID tags, and then calculates a group ID for each group. The relation between the group IDs of all the groups and the hashes of all the RFID tags can be expressed as follows:

$$
\left[\begin{array}{c}
\mathbf{g}_{1} \\
\vdots \\
\mathbf{g}_{m}
\end{array}\right]=\mathbf{A} \cdot\left[\begin{array}{c}
\mathbf{h}_{1} \\
\vdots \\
\mathbf{h}_{n}
\end{array}\right]
$$

where $m$ denotes the number of groups, $\mathbf{g}_{i}, i \in[m]$, is the group ID of the $i^{\text {th }}$ group, $n$ denotes the number of RFID tags, $\mathbf{h}_{j}, j \in[n]$, is the hash of the $j^{\text {th }}$ RFID tag, and $\mathbf{A}$ is an $m \times n\{0,1\}$-valued matrix, called a group generation matrix. The $(i, j)^{\text {th }}$ entry of $\mathbf{A}$ is equal to 1 if the $i^{\text {th }}$ group contains the $j^{\text {th }}$ RFID tag and is equal to 0 otherwise. To eliminate the requirement for the whole $\mathbf{A}$ upon decoding, grouping writes each group ID only to the memory of those RFID tags belonging to the group.

2) Decoding: By wirelessly interrogating RFID tags without errors, an interrogator can obtain the unique IDs of RFID tags and group IDs of their involved groups. Then it uses the HF to verify the integrity of a group. According to the verification results, (1) can be rearranged to the following form:

$$
\left[\begin{array}{c}
\mathrm{g}^{\mathrm{u}} \\
\hline \mathrm{g}^{\mathrm{c}} \\
\hline \mathrm{g}^{\mathrm{e}}
\end{array}\right]=\left[\begin{array}{c|c}
\mathbf{0} & \mathbf{A}^{\mathrm{um}} \\
\hline \mathbf{A}^{\mathrm{cr}} & \mathbf{0} \\
\hline \mathbf{A}^{\mathrm{er}} & \mathbf{A}^{\mathrm{em}}
\end{array}\right] \cdot\left[\begin{array}{c}
\mathbf{h}^{\mathbf{r}} \\
\hline \mathbf{h}^{\mathrm{m}}
\end{array}\right] .
$$

In (2), $\mathbf{g}^{\mathbf{u}}$ is the array of group IDs of the groups that are unknown because all the RFID tags belonging to these groups are missing, while $\mathbf{g}^{\mathbf{c}}$ and $\mathbf{g}^{\mathbf{e}}$ are those that preserve and lose their integrity, respectively. Likewise, $\mathbf{h}^{\mathbf{r}}$ and $\mathbf{h}^{\mathbf{m}}$ are the arrays of hashes of the RFID tags read by the interrogator and missing from the target collection, respectively. According to these arrays, $\mathbf{A}$ can be rearranged and separated into six submatrices $\mathbf{0}, \mathbf{A}^{\mathbf{c r}}, \mathbf{A}^{\mathrm{er}}, \mathbf{A}^{\mathbf{u m}}, \mathbf{0}$ and $\mathbf{A}^{\mathrm{em}}$. Among them, $\mathbf{A}^{\mathbf{u m}}$ and $\mathbf{A}^{\mathrm{em}}$ are not available upon decoding, as the information of $\mathbf{A}$ is distributed across RFID tags. According to (2), we can obtain:

$$
\begin{aligned}
& \mathbf{g}^{\mathbf{u}}=\mathbf{A}^{\mathbf{u m}} \mathbf{h}^{\mathbf{m}}, \\
& \mathbf{g}^{\mathbf{c}}=\mathbf{A}^{\mathbf{c r}} \mathbf{h}^{\mathbf{r}}, \\
& \mathbf{g}^{\mathbf{e}}=\mathbf{A}^{\mathrm{er}^{\mathbf{r}}} \mathbf{h}^{\mathbf{r}} \oplus \mathbf{A}^{\mathbf{e m}} \mathbf{h}^{\mathbf{m}} .
\end{aligned}
$$

(3) is useless because $\mathbf{g}^{\mathbf{u}}$ is unknown upon decoding, while (4) does not contain any information of missing RFID tags. The following useful result, however, can be obtained from (5):

$$
\mathbf{A}^{\mathbf{e m}} \mathbf{h}^{\mathbf{m}}=\mathbf{g}^{\mathbf{e}} \oplus \mathbf{A}^{\mathbf{e r}} \mathbf{h}^{\mathbf{r}} .
$$

Assuming $\mathbf{h}^{\mathbf{m}}$ is an array of the linearly independent hashes of missing RFID tags' unique IDs, the number of missing RFID tags is equal to the rank of $\mathbf{h}^{\mathbf{m}}$. $^{2}$ If $\mathbf{A}^{\mathrm{em}}$ can preserve the rank of $\mathbf{h}^{\mathbf{m}}$, the number of missing RFID tags can be accurately determined by calculating the rank of $\mathbf{A}^{\mathrm{em}} \mathbf{h}^{\mathrm{m}}$. Under this condition, because $\mathbf{g}^{\mathbf{e}}, \mathbf{A}^{\mathrm{er}}$ and $\mathbf{h}^{\mathbf{r}}$ are known, (6) implies that calculating the rank of $\mathbf{g}^{\mathbf{e}} \oplus \mathbf{A}^{\mathrm{er}} \mathbf{h}^{\mathbf{r}}$ determines the accurate number of missing RFID tags, regardless of the unavailability of $\mathbf{A}^{\mathrm{em}}$ and $\mathbf{h}^{\mathrm{m}}$.

\footnotetext{
${ }^{2}$ By the rank of $\mathbf{h}^{\mathbf{m}}$, we mean the rank of the binary matrix obtained from viewing the entries of $\mathbf{h}^{\mathbf{m}}$, i.e., the hashes of missing RFID tags, as binary row vectors.
} 


\section{B. Extended Grouping}

As seen in (6), the unknown submatrix $\mathbf{A}^{\mathbf{e m}}$ is the main difficulty for grouping to identify missing RFID tags. To overcome the problem, extended grouping writes additional information to the memory of RFID tags upon encoding.

1) Encoding: In addition to group IDs, extended grouping assigns a short ID to each RFID tag belonging to a target collection. The short ID is required to be unique within the target collection, and because the target collection size is generally much less than the maximum number of RFID tags, a portion of factory-programmed tag ID, e.g., 8 bits, is enough to practically serve the purpose. Therefore, each RFID tag in a group not only stores the group ID of this group, but also the short IDs of the other RFID tags belonging to this group. Additional difference from grouping is that the $\mathrm{HF}$ for extended grouping should be the trivial identity function, because the aim of extended grouping is to identify the unique IDs of missing RFID tags.

2) Decoding: Now an interrogator can retrieve $\mathbf{A}^{\mathbf{e m}}$ from the short IDs of missing RFID tags. As a result, upon decoding, (6) represents a set of linear equations with only $\mathbf{h}^{\mathbf{m}}$ unknown, and it defines a bipartite graph consisting of two sets of nodes: one of which represents the missing RFID tags, and the other the incomplete groups. In the bipartite graph, an edge connects a missing-RFID-tag node to an incompletegroup node if the missing RFID tag belongs to the incomplete group. While representing (6) as a bipartite graph, a simple alternative to solve (6) is by iterative message passing [13]. Clearly, another way to solve (6) is by Gaussian elimination, also called row reduction. In contrast to the linear decoding complexity of iterative message passing, Gaussian elimination, however, incurs higher decoding complexity.

\section{LDPC-Based Scheme}

The LDPC-based scheme uses the parity-check matrices of Gallager's LDPC codes as group generation matrices. The following gives the definition of LDPC codes.

Definition 1 ([13]): An ( $n, j, k)$ LDPC code is a code of block length $n$ with a parity-check matrix that has $j 1$ 's in each column, $k$ 1's in each row, and 0's elsewhere.

The following example illustrates the LDPC-based scheme.

Example 1: The following is an example of parity-check matrices of a $(9,2,3)$ Gallager's LDPC code [13]:

$$
\mathbf{A}=\left[\begin{array}{lllllllll}
1 & 1 & 1 & 0 & 0 & 0 & 0 & 0 & 0 \\
0 & 0 & 0 & 1 & 1 & 1 & 0 & 0 & 0 \\
0 & 0 & 0 & 0 & 0 & 0 & 1 & 1 & 1 \\
\hline 0 & 0 & 1 & 0 & 1 & 0 & 1 & 0 & 0 \\
1 & 0 & 0 & 0 & 0 & 0 & 0 & 1 & 1 \\
0 & 1 & 0 & 1 & 0 & 1 & 0 & 0 & 0
\end{array}\right] .
$$

The matrix in (7) is divided into two submatrices, each containing a single 1 in each column. As a group generation matrix, this matrix can divide a target collection of $n=9$ RFID tags into $m=6$ overlapping groups such that each group contains $k=3$ RFID tags and each RFID tag belongs to $j=2$ groups. Because the second submatrix is a random column permutation of the first, the columns in (7) are not necessarily linearly independent, e.g., columns 8 and 9. Suppose that the $8^{\text {th }}$ and $9^{\text {th }}$ RFID tags are missing. Therefore, the $3^{\text {rd }}$ and $5^{\text {th }}$ groups lose their integrity and, by reordering the rows of the matrix in (7), we can get

$$
\mathbf{A}=\left[\begin{array}{lllllll|ll}
1 & 1 & 1 & 0 & 0 & 0 & 0 & 0 & 0 \\
0 & 0 & 0 & 1 & 1 & 1 & 0 & 0 & 0 \\
0 & 0 & 1 & 0 & 1 & 0 & 1 & 0 & 0 \\
0 & 1 & 0 & 1 & 0 & 1 & 0 & 0 & 0 \\
\hline 0 & 0 & 0 & 0 & 0 & 0 & 1 & 1 & 1 \\
1 & 0 & 0 & 0 & 0 & 0 & 0 & 1 & 1
\end{array}\right]
$$

Clearly, from columns 8 and $9, \mathbf{A}^{\mathbf{e m}}=\left[\begin{array}{ll}1 & 1 \\ 1 & 1\end{array}\right]$ has rank equal to one, under which neither the rank of $\mathbf{h}^{\mathbf{m}}$ is preserved nor iterative message passing can identify the two missing RFID tags.

\section{SSF-Based Scheme}

We first give the definition of SSFs as follows.

Definition 2 ([14]): Let $r \leq n$. A family $\mathcal{F}$ of subsets of $[n]$ is said to be $(n, r)$-strongly-selective if, for any subset $\mathbb{X} \subset[n]$ of size (at most) $r$ and for every element $x \in \mathbb{X}$, there is a set $\mathbb{F}$ in $\mathcal{F}$ such that $\mathbb{F}$ selects $x$, i.e., $\mathbb{F} \cap \mathbb{X}=\{x\}$. Such a family is called an $(n, r)$-SSF.

The following example demonstrates the SSF-based scheme.

Example 2: The family $\mathcal{F}=\left\{\mathbb{F}_{1}, \ldots, \mathbb{F}_{6}\right\}$ of six subsets of $[9]$,

$$
\begin{array}{ll}
\mathbb{F}_{1}=\{1,2,3\}, & \mathbb{F}_{2}=\{4,5,6\}, \quad \mathbb{F}_{3}=\{7,8,9\}, \\
\mathbb{F}_{4}=\{1,6,8\}, & \mathbb{F}_{5}=\{2,4,9\}, \quad \mathbb{F}_{6}=\{3,5,7\},
\end{array}
$$

specifies a $(9,2)$-SSF. Let $\mathbb{F}_{i}, 1 \leq i \leq 6$, denote the set of nonzero positions of the $i^{\text {th }}$ row of a $\{0,1\}$-valued matrix $\mathbf{A}$, i.e.,

$$
\mathbf{A}=\left[\begin{array}{lllllllll}
1 & 1 & 1 & 0 & 0 & 0 & 0 & 0 & 0 \\
0 & 0 & 0 & 1 & 1 & 1 & 0 & 0 & 0 \\
0 & 0 & 0 & 0 & 0 & 0 & 1 & 1 & 1 \\
\hline 1 & 0 & 0 & 0 & 0 & 1 & 0 & 1 & 0 \\
0 & 1 & 0 & 1 & 0 & 0 & 0 & 0 & 1 \\
0 & 0 & 1 & 0 & 1 & 0 & 1 & 0 & 0
\end{array}\right]
$$

Similar to (7), the matrix in (9) consists of two submatrices, each containing a single one in each column. The first submatrix in (9) is also the same as that in (7). The second submatrix, however, is no longer a randomly chosen column permutation of the first. From columns 8 and $9, \mathbf{A}^{\mathbf{e m}}=\left[\begin{array}{ll}1 & 1 \\ 1 & 0 \\ 0 & 1\end{array}\right]$ has rank equal to two, under which the rank of $\mathbf{h}^{\mathbf{m}}$ is preserved and iterative message passing can identify the two missing RFID tags.

\section{Proposed SCHEME}

In this section, we first briefly present some background on the CRT, and then apply the CRT to construct group generation matrices for grouping and its extended counterpart. In addition, we present properties of the group generation matrices constructed based on the CRT. 


\section{A. Chinese Remainder Theorem (CRT)}

Let $q_{1}, \ldots, q_{v}$ denote $v$ positive integers that are relatively prime in pairs, i.e., $\operatorname{gcd}\left(q_{s}, q_{t}\right)=1, \forall s \neq t, 1 \leq s, t \leq v$, and $r_{1}, \ldots, r_{v}$ be integers such that $0 \leq r_{i}<q_{i}, 1 \leq i \leq v$. The CRT states that the following system of congruences

$$
\begin{aligned}
n & \equiv r_{1} \quad\left(\bmod q_{1}\right) \\
& \vdots \\
n & \equiv r_{v} \quad\left(\bmod q_{v}\right)
\end{aligned}
$$

has a unique solution modulo $\prod_{i=1}^{v} q_{i}$ [15].

By the CRT, a nonnegative integer $n$ is uniquely specified by the residues $r_{1}, \ldots, r_{v}$ of $n$ modulo $q_{1}, \ldots, q_{v}$, provided $n<\prod_{i=1}^{v} q_{i}$. Therefore, if we pick $j \geq v$ pairwise relatively prime integers $q_{1}<\cdots<q_{j}$ such that $n<\prod_{i=1}^{v} q_{i}$, then the residues of $n$ modulo $q_{1}, \ldots, q_{j}$ form a redundant representation of $n$, where we allow $j$ to equal $v$ for generality. The key property that allows for constructions of group generation matrices is that, by picking $j \geq v$ pairwise relatively prime integers $q_{1}<\cdots<q_{j}$, any two different nonnegative integers $n, n^{\prime}<\prod_{i=1}^{v} q_{i}$ have at least $j-v+1$ different residues modulo $q_{1}, \ldots, q_{j}$ [16].

\section{B. CRT-Based Scheme}

The use of CRT to construct group generation matrices starts by specifying the number $n$ of RFID tags in a target collection, the number $j$ of groups to which each RFID tag belongs, and a positive integer $v \leq j$. Then perform the following procedures, called the CRT-based scheme, to find a group generation matrix, which is denoted by $\mathbf{A}(n, j, v)$.

CRT-Based Scheme:

1) Select $j$ positive integers $q_{1}<\cdots<q_{j}$ that are pairwise relatively prime with the following two requirements:

$$
\begin{aligned}
q_{j} & \leq \frac{n}{2}, \\
\prod_{i=1}^{v} q_{i} & >n .
\end{aligned}
$$

2) For each integer $q_{i}, 1 \leq i \leq j$, construct a $\{0,1\}$-valued submatrix $\mathbf{A}(i)$ of size $q_{i} \times n$, where the $(s, t)^{\text {th }}$ entry is equal to 1 if

$$
t-1 \equiv s-1 \quad\left(\bmod q_{i}\right)
$$

is satisfied, and is equal to 0 otherwise.

3) Concatenate vertically submatrices $\mathbf{A}(i), 1 \leq i \leq j$, to form a group generation matrix $\mathbf{A}(n, j, v)$ of size $m \times n$, i.e.,

$$
\mathbf{A}(n, j, v)=\left[\begin{array}{c}
\mathbf{A}(1) \\
\hline \vdots \\
\hline \mathbf{A}(j)
\end{array}\right]
$$

where $m=\sum_{i=1}^{j} q_{i}$.

The following example illustrates the CRT-based scheme.

Example 3: For comparison with Examples 1 and 2, let us consider $n=9, j=2$ and $v=2$. Under these settings, the only possible choice of the pairwise relatively prime positive integers $\left(q_{1}, q_{2}\right)$ is $(3,4)$. The following $\{0,1\}$-valued matrix can then be constructed:

$$
\begin{aligned}
\mathbf{A}(9,2,2) & =\left[\begin{array}{l}
\mathbf{A}(1) \\
(2)
\end{array}\right] \\
& =\left[\begin{array}{lllllllll}
1 & 0 & 0 & 1 & 0 & 0 & 1 & 0 & 0 \\
0 & 1 & 0 & 0 & 1 & 0 & 0 & 1 & 0 \\
0 & 0 & 1 & 0 & 0 & 1 & 0 & 0 & 1 \\
\hline 1 & 0 & 0 & 0 & 1 & 0 & 0 & 0 & 1 \\
0 & 1 & 0 & 0 & 0 & 1 & 0 & 0 & 0 \\
0 & 0 & 1 & 0 & 0 & 0 & 1 & 0 & 0 \\
0 & 0 & 0 & 1 & 0 & 0 & 0 & 1 & 0
\end{array}\right]
\end{aligned}
$$

As a group generation matrix, $\mathbf{A}(9,2,2)$ in (13) can divide a target collection of $n=9$ RFID tags into $m=7$ overlapping groups such that each RFID tag belongs to $j=2$ groups. In contrast to the matrices in (7) and (9), one can see that the groups formed by $\mathbf{A}(9,2,2)$ in (13) can contain different numbers of RFID tags. From columns 8 and $9, \mathbf{A}^{\mathbf{e m}}(9,2,2)=$ $\left[\begin{array}{cc}1 & 0 \\ 0 & 1 \\ 0 & 1\end{array}\right]$

$\left[\begin{array}{ll}0 & 1 \\ 0 & 1\end{array}\right]$ has rank equal to two, under which the rank of $\mathbf{h}_{\mathbf{m}}$ is preserved and iterative message passing can identify the two missing RFID tags. Moreover, by the requirement in (10), each group contains at least two RFID tags. Also, one can see that the maximum value of the dot product of any two columns of $\mathbf{A}(9,2,2)$ in (13) is equal to one and that any two columns of $\mathbf{A}(9,2,2)$ in (13) contain a $2 \times 2$ identity submatrix.

\section{Properties of the Group Generation Matrices Constructed Based on the CRT}

The following gives the maximum value of the dot product of any two columns of a group generation matrix $\mathbf{A}(n, j, v)$ constructed based on the CRT.

Lemma 1: The maximum value of the dot product of any two columns of $\mathbf{A}(n, j, v)$ is equal to $v-1$.

Proof: Note that the rows and columns of a matrix are indexed from one and that a residue modulo $q_{i}$ must be in the range between 0 and $q_{i}-1$. By (12) in Step 2 of the CRT-based scheme, each integer $t \in[n]$ is associated with the residues of $t-1$ modulo $q_{1}, \ldots, q_{j}$. Then, by adding one to each of these residues, we obtain the nonzero positions in the $t^{\text {th }}$ columns of submatrices $\mathbf{A}(1), \ldots, \mathbf{A}(j)$ of $\mathbf{A}(n, j, v)$. Due to the restriction of the requirement in (11), one can see that any two different integers $t, t^{\prime} \in[n]$ are mapped to different positions in at least $j-v+1$ submatrices. As a result, the value of the dot product of any two columns of $\mathbf{A}(n, j, v)$ is at most equal to $j-(j-v+1)=v-1$.

The following result states that any small enough number of columns of $\mathbf{A}(n, j, v)$ contain an identity submatrix.

Theorem 1: In $\mathbf{A}(n, j, v)$, every $1+\left\lfloor\frac{j-1}{v-1}\right\rfloor$ columns contains a $\left(1+\left\lfloor\frac{j-1}{v-1}\right\rfloor\right) \times\left(1+\left\lfloor\frac{j-1}{v-1}\right\rfloor\right)$ identity submatrix.

Proof: Consider an arbitrary column of $\mathbf{A}(n, j, v)$, say column $t \in[n]$. By Lemma 1 , one can see that any $\left\lfloor\frac{j-1}{v-1}\right\rfloor$ columns of $\mathbf{A}(n, j, v)$ other than column $t$ can have 1 's in at most $\left\lfloor\frac{j-1}{v-1}\right\rfloor \cdot(v-1) \leq j-1$ same rows as column $t$. This indicates that column $t$ has at least a 1 in some row in which any other $\left\lfloor\frac{j-1}{v-1}\right\rfloor$ columns have 0's, as column $t$ has $j 1$ 's.

Remark 1: By Theorem 1, the CRT-based scheme guarantees that any number of missing RFID tags not greater than 
$1+\left\lfloor\frac{j-1}{v-1}\right\rfloor$ is determined accurately and that any subset of up to $1+\left\lfloor\frac{j-1}{v-1}\right\rfloor$ missing RFID tags is identified.

Remark 2: From the proof of Theorem 1, one can see that the componentwise OR of up to $\left\lfloor\frac{j-1}{v-1}\right\rfloor$ columns of $\mathbf{A}(n, j, v)$ covers only the columns, where a binary vector $\mathbf{x}$ is said to be covered in a binary vector $\mathbf{y}$ if and only if the componentwise OR of $\mathbf{x}$ and $\mathbf{y}$ is equal to $\mathbf{y}$. Therefore, the columns of a group generation matrix constructed based on the CRT form a superimposed code [17]. In combinatorics, superimposed codes are known as cover-free families [18] and also closely connected to nonadaptive group testing [19]. Further, it should be noted from Theorem 1 and Definition 2 that the CRT-based scheme gives an explicit construction of SSFs.

The following gives the total memory consumptions of grouping and its extended counterpart based on $\mathbf{A}(n, j, v)$, denoted by $M^{g}(\mathbf{A}(n, j, v))$ and $M^{e}(\mathbf{A}(n, j, v))$, respectively, where the total memory consumption is the total memory usage of group IDs and short IDs, not including unique IDs.

Theorem 2: The total memory consumption of grouping based on $\mathbf{A}(n, j, v)$ with $q_{1}<\cdots<q_{j}$ is equal to $M^{g}(\mathbf{A}(n, j, v))=n \cdot j \cdot c_{1}$, while that of extended grouping is equal to

$$
\begin{aligned}
& M^{e}(\mathbf{A}(n, j, v))= \\
& n \cdot j \cdot c_{2}+\sum_{i=1}^{j}\left[\left(n \bmod q_{i}\right) \cdot\left(\left\lfloor\frac{n}{q_{i}}\right\rfloor+1\right) \cdot\left\lfloor\frac{n}{q_{i}}\right\rfloor+\right. \\
& \left.\left.\quad\left(q_{i}-\left(n \bmod q_{i}\right)\right) \cdot\left\lfloor\frac{n}{q_{i}}\right\rfloor \cdot\left(\left\lfloor\frac{n}{q_{i}}\right\rfloor-1\right)\right] \cdot c_{3},(14)\right)
\end{aligned}
$$

where $c_{1}$ and $c_{2}$ are the memory consumptions of a group ID in grouping and its extended counterpart, respectively, ${ }^{3}$ and $c_{3}$ is the memory consumption of a short ID.

Proof: The total memory consumption of grouping based on $\mathbf{A}(n, j, v)$ follows from the property that $\mathbf{A}(n, j, v)$ has $n$ columns, in each of which there are $j 1$ 's. In addition to the total memory consumption $n \cdot j \cdot c_{2}$ used to store group IDs, extended grouping consumes additional memory for storing short IDs. In submatrix $\mathbf{A}(i), 1 \leq i \leq j$, the rows can be divided into two parts: one of which is the first $\left(n \bmod q_{i}\right)$ rows, each containing $\left\lfloor\frac{n}{q_{i}}\right\rfloor+11$ 's, and the other the remaining $q_{i}-\left(n \bmod q_{i}\right)$ rows, each containing $\left\lfloor\frac{n}{q_{i}}\right\rfloor 1$ 's. In the former part, each RFID tag in a group requires to store $\left\lfloor\frac{n}{q_{i}}\right\rfloor$ short IDs of the other RFID tags belonging to the same group, while in the latter part, each RFID tag in a group requires to store $\left\lfloor\frac{n}{q_{i}}\right\rfloor-1$ short IDs. Summing up these memory requirements, we obtain the total memory consumption of extended grouping, as given by (14).

\section{On the Choice of the Pairwise Relatively Prime Integers}

In Step 1 of the CRT-based scheme, there might be many different choices of the pairwise relatively prime integers $q_{i}$ 's. The optimal choice of $q_{i}$ 's depends on the performance criterion employed. For example, because minimizing the number of groups can reduce decoding complexity, a possible

\footnotetext{
${ }^{3}$ Grouping and its extended counterpart might have different memory consumptions of a group ID as they can employ different HFs.
}

choice option that we will consider is to minimize the number $m$ of rows of $\mathbf{A}(n, j, v)$, i.e., to select the smallest consecutive pairwise relatively prime integers. On the other hand, by Theorem 2, the total memory consumption of extended grouping heavily depends on the choice of $q_{i}$ 's. Therefore, another intuitive choice that will also be considered later is to select $q_{i}$ 's as the largest consecutive pairwise relatively prime integers such that the total memory consumption of extended grouping is minimized. Although these two selection policies might not always lead to optimal performance, they represent the two extreme cases of the choice of $q_{i}$ 's and can reveal tradeoffs among different choices of $q_{i}$ 's, as will be seen in Section V.

\section{Decoding Guarantees And Theoretical ANALYSES OF ERROR RATES}

By examining any three columns of $\mathbf{A}(9,2,2)$ in (13), it is not hard to see that the decoding guarantees of grouping and its extended counterpart based on $\mathbf{A}(9,2,2)$ in (13) are at least equal to three. Therefore, the decoding guarantees determined by Theorem 1 are not exact results and, actually, they are just lower bounds. In this section, we characterize the key objects needed to study decoding guarantees of grouping and its extended counterpart, which enable theoretical analyses of error rates. Relationships between the key objects are also presented.

Below, we first give the definition of error rates.

Definition 3: The error rate is the probability of decoding failures, where a decoding failure means that, after finishing decoding, the number of missing RFID tags is not determined accurately or not all the missing RFID tags can be identified.

\section{A. Decoding Guarantee and Error Rate of Grouping}

Assume that the hashes of RFID tags are linearly independent. Then we have

$$
\begin{aligned}
\operatorname{rank}\left(\mathbf{A}^{\mathbf{e m}}\right) & =\operatorname{rank}\left(\mathbf{A}^{\mathbf{e m}} \mathbf{h}^{\mathbf{m}}\right) \\
& \leq \operatorname{rank}\left(\mathbf{h}^{\mathbf{m}}\right),
\end{aligned}
$$

where equality holds when the columns of $\mathbf{A}^{\mathbf{e m}}$ are linearly independent. Therefore, for grouping, decoding failures occur when $\operatorname{rank}\left(\mathbf{A}^{\mathbf{e m}}\right)<\operatorname{rank}\left(\mathbf{h}^{\mathbf{m}}\right)$. In other words, grouping cannot accurately determine the number of missing RFID tags when $\mathbf{A}^{\mathrm{em}}$ does not have full column rank. To characterize decoding failures of grouping, we introduce the notion of rankdeficient sets as follows.

Definition 4: A rank-deficient set of a group generation matrix $\mathbf{A}$ is a subset of column indices of $\mathbf{A}$ such that the submatrix $\mathbf{A}^{\mathbf{e m}}$ from the corresponding columns of $\mathbf{A}$ does not have full column rank.

It is worth pointing out that a rank-deficient set cannot be empty, as the rank of an empty matrix is defined to be equal to zero. By Definition 4, one can see that the decoding guarantee of grouping based on $\mathbf{A}$, denoted by $N^{g}(\mathbf{A})$, is equal to the smallest size of rank-deficient sets minus one. For example, for $\mathbf{A}(9,2,2)$ in (13), $\{1,3,4,7\}$ is a smallest rank-deficient set, thereby leading to $N^{g}(\mathbf{A}(9,2,2))=3$. Assuming $n$ RFID tags are grouped based on $\mathbf{A}$ and each RFID tag is missing 
independently of any other and with equal probability, the error rate of grouping when $i$ RFID tags are missing is given by

$$
E^{g}(\mathbf{A}, i)=\frac{R(\mathbf{A}, i)}{\left(\begin{array}{c}
n \\
i
\end{array}\right)},
$$

where $R(\mathbf{A}, i)$ is the number of rank-deficient sets of size $i$ of $\mathbf{A}$.

Remark 3: Recall that the minimum distance of a linear code can be defined as the minimum nonzero number of columns of a parity-check matrix of the linear code that are not linearly independent [20]. Suppose in Definition 4 that the submatrix $\mathbf{A}^{\mathrm{em}}$ is replaced with the group generation matrix A. Then one can see that the smallest size of rank-deficient sets of $\mathbf{A}$ is equal to the minimum distance of a linear code with $\mathbf{A}$ as its parity-check matrix.

In general, it is very hard to give a full characterization of rank-deficient sets of an arbitrary group generation matrix $\mathbf{A}$ due to its combinatorial nature. Therefore, it is also difficult to determine the decoding guarantee of grouping based on $\mathbf{A}$. Some results on the decoding guarantee of grouping based on $\mathbf{A}(n, j, v)$, however, can be obtained. The following first gives the decoding guarantee of grouping based on $\mathbf{A}(n, 2,2)$.

Theorem 3: For $\mathbf{A}(n, 2,2)$ with $q_{1}<q_{2} \leq \frac{n}{2}$, the decoding guarantee of grouping is

$$
N^{g}(\mathbf{A}(n, 2,2))= \begin{cases}2, & \text { if } 2 q_{2} \leq n<3 q_{1} \\ 3, & \text { if } 3 q_{1} \leq n<q_{1} q_{2} .\end{cases}
$$

Proof: If $2 q_{2} \leq n<3 q_{1}$, by Theorem 1 , it then suffices to display a rank-deficient set of size three for $\mathbf{A}(n, 2,2)$. Note first that, given $2 q_{2} \leq n<3 q_{1}$, both submatrices $\mathbf{A}(1)$ and $\mathbf{A}(2)$ of $\mathbf{A}(n, 2,2)$ contain some rows with exactly two 1 's. Then consider the set of column indices $\mathbb{M}_{1}=$ $\left\{q_{1}, 2 q_{1} \bmod q_{2}, 2 q_{1}\right\}$. By Step 2 of the CRT-based scheme, columns $2 q_{1}$ and $q_{1}$ have nonzero entries in the same row of $\mathbf{A}(1)$, which contains exactly two 1's. Similarly, columns $2 q_{1}$ and $2 q_{1} \bmod q_{2}$ have nonzero entries in the same row of $\mathbf{A}(2)$, which also contains exactly two 1's. It is then easily verified that the submatrix $\mathbf{A}^{\mathbf{e m}}(n, 2,2)$ from the columns of $\mathbf{A}(n, 2,2)$ indexed by $\mathbb{M}_{1}$ has a zero column, which corresponds to column $2 q_{1}$ of $\mathbf{A}(n, 2,2)$. Therefore, $\mathbb{M}_{1}$ is a rank-deficient set.

On the other hand, if $3 q_{1} \leq n<q_{1} q_{2}$, we first need to show that there is no rank-deficient set of size three. Suppose, to the contrary, that there is a rank-deficient set of size three for $\mathbf{A}(n, 2,2)$. In $\mathbf{A}^{\mathbf{e m}}(n, 2,2)$ from the columns of $\mathbf{A}(n, 2,2)$ indexed by the rank-deficient set of size three, by Definition 4, there must exist one, two or three columns, whose componentwise XOR gives a zero column vector. With $3 q_{1} \leq n<q_{1} q_{2}$, each row of $\mathbf{A}(1)$ has at least three 1's. As a result, $\mathbf{A}^{\mathbf{e m}}(n, 2,2)$ from the columns of $\mathbf{A}(n, 2,2)$ indexed by the rank-deficient set of size three cannot have a zero column. By Lemma 1, any two columns of $\mathbf{A}^{\mathbf{e m}}(n, 2,2)$ cannot be XORed componentwise to a zero column vector. Then the componentwise XOR of all the columns of $\mathbf{A}^{\mathbf{e m}}(n, 2,2)$ must be a zero column vector. Clearly, [ $\left.\begin{array}{lll}1 & 1 & 1\end{array}\right],\left[\begin{array}{lll}1 & 0 & 0\end{array}\right],\left[\begin{array}{lll}0 & 1 & 0\end{array}\right]$ and $\left[\begin{array}{lll}0 & 0 & 1\end{array}\right]$ cannot be rows of $\mathbf{A}^{\mathbf{e m}}(n, 2,2)$, as each of them cannot be XORed to zero. Although [ [ $\left.\begin{array}{lll}1 & 1 & 0\end{array}\right],\left[\begin{array}{lll}1 & 0 & 1\end{array}\right]$ and $\left[\begin{array}{lll}0 & 1 & 1\end{array}\right]$ can be XORed to zero, by Lemma 1 , they must be accompanied

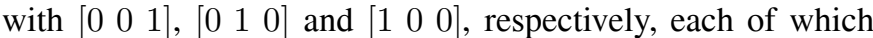
cannot be XORed to zero. Therefore, $\mathbf{A}^{\mathbf{e m}}(n, 2,2)$ cannot contain either $\left[\begin{array}{lll}1 & 1 & 0\end{array}\right],\left[\begin{array}{lll}1 & 0 & 1\end{array}\right]$ or $\left[\begin{array}{lll}0 & 1 & 1\end{array}\right]$, and the only possible row that can appear in $\mathbf{A}^{\mathbf{e m}}(n, 2,2)$ is $\left[\begin{array}{lll}0 & 0 & 0\end{array}\right]$, resulting in a contradiction. Next, we display a rank-deficient set of size four for $\mathbf{A}(n, 2,2)$. Consider the set of column indices $\mathbb{M}_{2}=\left\{n-q_{1}-q_{2}, n-q_{2}, n-q_{1}, n\right\}$. Because

$$
\begin{aligned}
n-q_{1}-q_{2} & =n-q_{2} \quad\left(\bmod q_{1}\right), \\
n-q_{1} & =n \quad\left(\bmod q_{1}\right), \\
n-q_{1}-q_{2} & =n-q_{1} \quad\left(\bmod q_{2}\right), \text { and } \\
n-q_{2} & =n \quad\left(\bmod q_{2}\right),
\end{aligned}
$$

then up to a column permutation, the columns of $\mathbf{A}(n, 2,2)$ indexed by $\mathbb{M}_{2}$, excluding zero rows, must be of the form $\left[\begin{array}{llll}1 & 1 & 0 & 0 \\ 0 & 0 & 1 & 1 \\ 0 & 0 & 1 & 0 \\ 0 & 1 & 0 & 0\end{array}\right]$. Therefore, the componentwise XOR of all the columns of $\mathbf{A}^{\mathbf{e m}}(n, 2,2)$ from the columns of $\mathbf{A}(n, 2,2)$ indexed by $\mathbb{M}_{2}$ is a zero column vector and $\mathbb{M}_{2}$ is a rankdeficient set. This completes the proof.

We next present results on the decoding guarantee of grouping based on $\mathbf{A}(n, 3,2)$.

Theorem 4: For $\mathbf{A}(n, 3,2)$ constructed with $q_{1}<q_{2}<$ $q_{3} \leq \frac{n}{2}$, the decoding guarantee of grouping is

$$
N^{g}(\mathbf{A}(n, 3,2)) \begin{cases}=3, & \text { if } 2 q_{3} \leq n<3 q_{1} \\ =4, & \text { if } 3 q_{1} \leq n<\min \left(4 q_{1}, 2 q_{2}+q_{1}\right) \\ \geq 5, & \text { if } \min \left(4 q_{1}, 2 q_{2}+q_{1}\right) \leq n<q_{1} q_{2} .\end{cases}
$$

Proof: If $2 q_{3} \leq n<3 q_{1}$, the proof is similar to that of Theorem 3. By Theorem 1, it is enough to display a rankdeficient set of size four for $\mathbf{A}(n, 3,2)$. Consider the set of column indices $\mathbb{M}_{3}=\left\{q_{1}, 2 q_{1} \bmod q_{2}, 2 q_{1} \bmod q_{3}, 2 q_{1}\right\}$. Columns $2 q_{1}$ and $q_{1}$, columns $2 q_{1}$ and $2 q_{1} \bmod q_{2}$, and columns $2 q_{1}$ and $2 q_{1} \bmod q_{3}$ have nonzero entries in the same rows of $\mathbf{A}(1), \mathbf{A}(2)$, and $\mathbf{A}(3)$, respectively, each of which contains exactly two 1's. Therefore, $\mathbf{A}^{\mathbf{e m}}(n, 3,2)$ from the columns of $\mathbf{A}(n, 3,2)$ indexed by $\mathbb{M}_{3}$ has a zero column, which corresponds to column $2 q_{1}$ of $\mathbf{A}(n, 3,2)$, and, therefore, $\mathbb{M}_{3}$ is a rank-deficient set.

If $3 q_{1} \leq n<\min \left(4 q_{1}, 2 q_{2}+q_{1}\right)$, we first show that there is no rank-deficient set of size four for $\mathbf{A}(n, 3,2)$. Suppose, to the contrary, that there is a rank-deficient set of size four for $\mathbf{A}(n, 3,2)$. Following a similar idea as the proof of Theorem 3, one can see that the submatrix $\mathbf{A}^{\mathbf{e m}}(n, 3,2)$ from the columns of $\mathbf{A}(n, 3,2)$ indexed by the rank-deficient set of size four cannot have a zero column, and any two or three columns of $\mathbf{A}^{\mathbf{e m}}(n, 3,2)$ cannot be XORed componentwise to a zero column vector. The only possibility for the claim to hold is that the componentwise XOR of all the columns of $\mathbf{A}^{\mathbf{e m}}(n, 3,2)$ is a zero column vector, which implies that $\mathbf{A}^{\mathbf{e m}}(n, 3,2)$ consists of rows with four or two 1's. Clearly, $\mathbf{A}^{\mathbf{e m}}(n, 3,2)$ cannot contain a row with four 1's, as it must be accompanied with at least one row with a single 1 , which is a contradiction. On the other hand, if $\mathbf{A}^{\mathbf{e m}}(n, 3,2)$ consists of rows with exactly two 1's, up to a column permutation, the columns of $\mathbf{A}(n, 3,2)$ indexed by the rank-deficient set of size four must contain a four-column submatrix of the form $\left[\begin{array}{llll}1 & 1 & 0 & 0 \\ 0 & 0 & 1 & 1 \\ 0 & 0 & 1 & 0 \\ 0 & 1 & 0 & 1 \\ 0 & 0 & 0 & 1 \\ 0 & 1 & 1 & 0\end{array}\right]$. Without loss of 
generality, the four-column submatrix is assumed to consist of three $2 \times 4$ submatrices from $\mathbf{A}(1), \mathbf{A}(2)$ and $\mathbf{A}(3)$, respectively, from top to bottom. According to the top two submatrices, the rank-deficient set of size four must be of the form $\left\{t, t+k_{1} q_{1}, t+k_{2} q_{2}, t+k_{1} q_{1}+k_{2} q_{2}+k_{12} q_{1} q_{2}\right\}$, where $t \in[n], k_{1} \geq 1, k_{2} \geq 1$ and $k_{12} \geq 0$. For $\mathbf{A}(n, 3,2), n$ must be less than $q_{1} q_{2}$, which implies $k_{12}=0$. Then according to the bottom submatrix, we have

$$
\begin{aligned}
t & \equiv t+k_{1} q_{1}+k_{2} q_{2} \quad\left(\bmod q_{3}\right), \\
t+k_{1} q_{1} & \equiv t+k_{2} q_{2} \quad\left(\bmod q_{3}\right) .
\end{aligned}
$$

Subtracting (16) from (17) yields

$$
\begin{aligned}
k_{1} q_{1} & \equiv-k_{1} q_{1} \quad\left(\bmod q_{3}\right), \\
q_{1} & \equiv-q_{1} \quad\left(\bmod q_{3}\right), \\
q_{1} & =q_{3}-q_{1}, \\
q_{3} & =2 q_{1},
\end{aligned}
$$

which is in contradiction to the pairwise relatively primality of $q_{i}$ 's. We next display a rank-deficient set of size five. Consider the set of column indices $\mathbb{M}_{4}=\left\{2 q_{2}-2 q_{1}, 2 q_{2}-\right.$ $\left.q_{1}, q_{2}, 2 q_{2} \bmod q_{3}, 2 q_{2}\right\}$. It is easily verified that the submatrix $\mathbf{A}^{\mathbf{e m}}(n, 3,2)$ from the columns of $\mathbf{A}(n, 3,2)$ indexed by $\mathbb{M}_{4}$ must contain a zero column, which corresponds to column $2 q_{2}$ of $\mathbf{A}(n, 3,2)$. Therefore, $\mathbb{M}_{4}$ is a rank-deficient set.

If $\min \left(4 q_{1}, 2 q_{2}+q_{1}\right) \leq n<q_{1} q_{2}$, we first show that there is no rank-deficient set of size five for $\mathbf{A}(n, 3,2)$. Suppose, to the contrary, that there is a rank-deficient set of size five for $\mathbf{A}(n, 3,2)$. With $\min \left(4 q_{1}, 2 q_{2}+q_{1}\right) \leq n<q_{1} q_{2}$, to examine whether or not there is a zero column in the submatrix $\mathbf{A}^{\mathbf{e m}}(n, 3,2)$ from the columns of $\mathbf{A}(n, 3,2)$ indexed by the rank-deficient set of size five, it suffices to consider column $2 q_{2}$ of $\mathbf{A}(n, 3,2)$. Clearly, the row of $\mathbf{A}(1)$ that intersects column $2 q_{2}$ with a nonzero entry contains at least four 1 's. This indicates that $\mathbf{A}^{\mathbf{e m}}(n, 3,2)$ cannot contain a zero column. Moreover, one can see that any two, three or four columns of $\mathbf{A}^{\mathbf{e m}}(n, 3,2)$ cannot be XORed componentwise to a zero column vector. Then, the componentwise XOR of all the columns of $\mathbf{A}^{\mathbf{e m}}(n, 3,2)$ must be a zero column vector, indicating that $\mathbf{A}^{\mathbf{e m}}(n, 3,2)$ consists of rows with four or two 1 's. If $\mathbf{A}^{\mathbf{e m}}(n, 3,2)$ contains a row with four 1's, then it must also contain a row with a single 1 , which is a contradiction. On the other hand, if $\mathbf{A}^{\mathbf{e m}}(n, 3,2)$ contains a row with two 1 's, then up to a column permutation, we may assume that the columns of $\mathbf{A}(n, 3,2)$ indexed by the rank-deficient set of size five contain a submatrix of the form $\left[\begin{array}{lllll}1 & 1 & 0 & 0 & 0 \\ 0 & 0 & 1 & 1 & 1\end{array}\right]$ from $\mathbf{A}(1)$. Under this condition, the submatrix from $\mathbf{A}(2)$ or $\mathbf{A}(3)$ to be contained in the columns of $\mathbf{A}(n, 3,2)$ indexed by the rank-deficient set of size five must contain a row with a single 1 , resulting in a contradiction. This concludes the proof.

The following gives the decoding guarantee of grouping based on $\mathbf{A}(n, 3,3)$ when $n$ is sufficiently large.

Theorem 5: For $\mathbf{A}(n, 3,3)$ with $q_{1}<q_{2}<q_{3} \leq \frac{n}{2}$, if $1+$ $q_{1} q_{2}+q_{3} \leq n<q_{1} q_{2} q_{3}$, the decoding guarantee of grouping is $N^{g}(\mathbf{A}(n, 3,3))=3$.

Proof: If $1+q_{1} q_{2}+q_{3} \leq n<q_{1} q_{2} q_{3}$, by Theorem 1 , there is no rank-deficient set of size two for $\mathbf{A}(n, 3,3)$. In addition, by the reasoning used in the proof of Theorem 3 , it is easy to see that there is no rank-deficient set of size three for $\mathbf{A}(n, 3,3)$. Then it remains to display a rank-deficient set of size four for $\mathbf{A}(n, 3,3)$. It is easily verified that $\mathbb{M}_{5}=\left\{1,1+q_{3}, 1+\right.$ $\left.q_{1} q_{2}, 1+q_{1} q_{2}+q_{3}\right\}$ is a rank-deficient set of size four.

\section{B. Decoding Guarantee and Error Rate of Extended Grouping}

It has been demonstrated in [21] and [22] that decoding failures of iterative message passing decoding over erasure channels are characterized by certain structures, called deadend sets, of a parity-check matrix of a code. However, for the purpose of our exposition, we use a slightly modified version of dead-end sets, which is given below.

Definition 5: A dead-end set of a group generation matrix $\mathbf{A}$ is a subset of column indices of $\mathbf{A}$ such that the submatrix $\mathbf{A}^{\mathbf{e m}}$ from the corresponding columns of $\mathbf{A}$ has a submatrix consisting of its columns which does not contain a row of weight one.

It should be noted from Definition 5 that a dead-end set can be empty. By characterizing dead-end sets, one can see that, whenever the set of column indices of $\mathbf{A}$ corresponding to the missing RFID tags is a nonempty dead-end set, not all the missing RFID tags can be identified. Therefore, the decoding guarantee of extended grouping based on $\mathbf{A}$, denoted by $N^{e}(\mathbf{A})$, is equal to the smallest size of nonempty dead-end sets minus one. Taking $\mathbf{A}(9,2,2)$ in (13) as an example again, one can see that $\{1,3,4,7\}$ is a nonempty smallest dead-end set, indicating $N^{e}(\mathbf{A}(9,2,2))=3$. Similar to the error rate of grouping in (15), one can also see that the error rate of extended grouping based on $\mathbf{A}$ when $i$ out of $n$ RFID tags are missing is given by

$$
E^{e}(\mathbf{A}, i)=\frac{D(\mathbf{A}, i)}{\left(\begin{array}{c}
n \\
i
\end{array}\right)},
$$

where $D(\mathbf{A}, i)$ is the number of dead-end sets of size $i$ of $\mathbf{A}$.

Remark 4: The main difference between Definition 5 and that in [21] is the need to investigate the submatrix $\mathbf{A}^{\mathbf{e m}}$ of the group generation matrix $\mathbf{A}$. This is because information of some rows of $\mathbf{A}$ may not be available upon decoding. Therefore, similarly to Remark 3, if, in Definition 5, $\mathbf{A}^{\mathbf{e m}}$ is replaced with $\mathbf{A}$, the smallest size of dead-end sets of $\mathbf{A}$ is equal to the stopping distance of a linear code with $\mathbf{A}$ as its parity-check matrix [21], [22].

Much like rank-deficient sets, it is very challenging to give a full characterization of dead-end sets of an arbitrary group generation matrix A [23]. Therefore, it is also challenging to determine the decoding guarantee of extended grouping based on A. Similar to [24] and [25], some results on the decoding guarantee of extended grouping based on $\mathbf{A}(n, j, v)$, however, can be obtained. The following first gives the decoding guarantee of extended grouping based on $\mathbf{A}(n, 2,2)$.

Theorem 6: For $\mathbf{A}(n, 2,2)$ with $q_{1}<q_{2} \leq \frac{n}{2}$, the decoding guarantee of extended grouping is

$$
N^{e}(\mathbf{A}(n, 2,2))= \begin{cases}2, & \text { if } 2 q_{2} \leq n<3 q_{1} \\ 3, & \text { if } 3 q_{1} \leq n<q_{1} q_{2} .\end{cases}
$$

Proof: We follow the same procedure as in the proof of Theorem 3. If $2 q_{2} \leq n<3 q_{1}$, it is enough to see that $\mathbb{M}_{1}=$ 
$\left\{q_{1}, 2 q_{1} \bmod q_{2}, 2 q_{1}\right\}$ is a dead-end set. On the other hand, if $3 q_{1} \leq n<q_{1} q_{2}$, we first show that there is no dead-end set of size three. On the contrary, suppose that there is a deadend set of size three for $\mathbf{A}(n, 2,2)$. With $3 q_{1} \leq n<q_{1} q_{2}$, the submatrix $\mathbf{A}^{\mathbf{e m}}(n, 2,2)$ from the columns of $\mathbf{A}(n, 2,2)$ indexed by the dead-end set of size three cannot have a zero column. Then by Definition $5, \mathbf{A}^{\mathbf{e m}}(n, 2,2)$ cannot contain $\left[\begin{array}{lll}1 & 0 & 0\end{array}\right],\left[\begin{array}{lll}0 & 1 & 0\end{array}\right]$ and $\left[\begin{array}{lll}0 & 0 & 1\end{array}\right]$. In addition, according to the structure of $\mathbf{A}(n, 2,2),\left[\begin{array}{lll}1 & 1 & 0\end{array}\right],\left[\begin{array}{lll}1 & 0 & 1\end{array}\right]$ and $\left[\begin{array}{lll}0 & 1 & 1\end{array}\right]$ cannot be rows of $\mathbf{A}^{\mathbf{e m}}(n, 2,2)$, as they must be accompanied with $\left[\begin{array}{lll}0 & 0 & 1\end{array}\right],\left[\begin{array}{lll}0 & 1 & 0\end{array}\right]$ and $\left[\begin{array}{lll}1 & 0 & 0\end{array}\right]$, respectively. Then the only possible row that can be contained in $\mathbf{A}^{\mathbf{e m}}(n, 2,2)$ is $\left[\begin{array}{lll}1 & 1 & 1\end{array}\right]$. If $\mathbf{A}^{\mathbf{e m}}(n, 2,2)$ contains [ $\left.\begin{array}{lll}1 & 1 & 1\end{array}\right]$ as a row, by Lemma 1 , it must contain $\left[\begin{array}{lll}1 & 0 & 0\end{array}\right],\left[\begin{array}{lll}0 & 1 & 0\end{array}\right]$ and $\left[\begin{array}{lll}0 & 0 & 1\end{array}\right]$, which is a contradiction. It remains to display a dead-end set of size four for $\mathbf{A}(n, 2,2)$. It is easy to verify that $\mathbb{M}_{2}=\left\{n-q_{1}-q_{2}, n-q_{2}, n-q_{1}, n\right\}$ is a dead-end set.

Similar to Theorem 4, the following gives results on the decoding guarantee of extended grouping based on $\mathbf{A}(n, 3,2)$.

Theorem 7: For $\mathbf{A}(n, 3,2)$ with $q_{1}<q_{2}<q_{3} \leq \frac{n}{2}$, the decoding guarantee of extended grouping is

$$
N^{e}(\mathbf{A}(n, 3,2)) \begin{cases}=3, & \text { if } 2 q_{3} \leq n<3 q_{1} \\ =4, & \text { if } 3 q_{1} \leq n<\min \left(4 q_{1}, 2 q_{2}+q_{1}\right) \\ \geq 5, & \text { if } \min \left(4 q_{1}, 2 q_{2}+q_{1}\right) \leq n<q_{1} q_{2} .\end{cases}
$$

Proof: We also follow the same procedure as in the proof of Theorem 4. If $2 q_{3} \leq n<3 q_{1}$, it is enough to see that $\mathbb{M}_{3}=\left\{q_{1}, 2 q_{1} \bmod q_{2}, 2 q_{1} \bmod q_{3}, 2 q_{1}\right\}$ is a deadend set. If $3 q_{1} \leq n<\min \left(4 q_{1}, 2 q_{2}+q_{1}\right)$, we first show that there is no dead-end set of size four for $\mathbf{A}(n, 3,2)$. Suppose, to the contrary, that there is a dead-end set of size four for $\mathbf{A}(n, 3,2)$. Following a similar idea as the proof of Theorem 4, the submatrix $\mathbf{A}^{\mathbf{e m}}(n, 3,2)$ from the columns of $\mathbf{A}(n, 3,2)$ indexed by the dead-end set of size four cannot have a zero column and can consist of rows with only two 1's. Therefore, up to a column permutation, the columns of $\mathbf{A}(n, 3,2)$ indexed by the dead-end set of size four must contain a four-column submatrix of the form $\left[\begin{array}{llll}1 & 1 & 0 & 0 \\ 0 & 0 & 1 & 1 \\ 1 & 0 & 1 & 0 \\ 0 & 1 & 1 & 0\end{array}\right.$

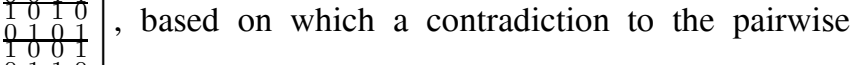
relatively primality of $q_{i}$ 's follows. Then it is easily verified that $\mathbb{M}_{4}=\left\{2 q_{2}-2 q_{1}, 2 q_{2}-q_{1}, q_{2}, 2 q_{2} \bmod q_{3}, 2 q_{2}\right\}$ is a dead-end set of five. If $\min \left(4 q_{1}, 2 q_{2}+q_{1}\right) \leq n<q_{1} q_{2}$, we show that there is no dead-end set of size five for $\mathbf{A}(n, 3,2)$. Suppose, to the contrary, that there is a dead-end set of size five for $\mathbf{A}(n, 3,2)$. With $\min \left(4 q_{1}, 2 q_{2}+q_{1}\right) \leq n<q_{1} q_{2}$, $\mathbf{A}^{\mathbf{e m}}(n, 3,2)$ from the columns of $\mathbf{A}(n, 3,2)$ indexed by the dead-end set of size five cannot contain a zero column. Then up to a column permutation, the columns of $\mathbf{A}(n, 3,2)$ indexed by the dead-end set of size five contain a submatrix of the form $\left[\begin{array}{lllll}1 & 1 & 0 & 0 & 0 \\ 0 & 0 & 1 & 1 & 1\end{array}\right]$. Under this condition, the columns of $\mathbf{A}(n, 3,2)$ indexed by the dead-end set of size five must contain a row with a single 1 , resulting in a contradiction. This concludes the proof.

Similar to Theorem 8, the following gives the decoding guarantee of extended grouping based on $\mathbf{A}(n, 3,3)$ when $n$ is sufficiently large.
Theorem 8: For $\mathbf{A}(n, 3,3)$ with $q_{1}<q_{2}<q_{3} \leq \frac{n}{2}$, if $1+$ $q_{1} q_{2}+q_{3} \leq n<q_{1} q_{2} q_{3}$, the decoding guarantee of extended grouping is $N^{e}(\mathbf{A}(n, 3,3))=3$.

Proof: If $1+q_{1} q_{2}+q_{3} \leq n<q_{1} q_{2} q_{3}$, by Theorem 1, there is no dead-end set of size two. By the reasoning used in the proof of Theorem 6, one can also see that there is no deadend set of size three. Then it is readily verified that $\mathbb{M}_{5}=$ $\left\{1,1+q_{3}, 1+q_{1} q_{2}, 1+q_{1} q_{2}+q_{3}\right\}$ is a dead-end set of size four. This concludes the proof.

\section{Relationships Between Rank-Deficient and Dead-End Sets}

One can see that Theorems 3-5 are similar to Theorems 68 and that their proofs are very similar. In addition, it is clear from the proofs of Theorems 3-8 that a rank-deficient set is a dead-end set. This does not happen by chance, as shown below.

Theorem 9: For any group generation matrix $\mathbf{A}$, a rankdeficient set is a dead-end set, but the converse is not always true.

Proof: Given a rank-deficient set $\mathbb{M}$ of $\mathbf{A}$, there exists a nontrivial linear combination of all the columns of $\mathbf{A}^{\mathbf{e m}}$ from the columns of $\mathbf{A}$ indexed by $\mathbb{M}$ that gives a zero column vector. In other words, there exists a set of binary numbers, $\left\{a_{1}, \ldots, a_{|\mathbb{M}|}\left|a_{i} \in\{0,1\}, 1 \leq i \leq\right| \mathbb{M} \mid\right\}$, not all zero, such that

$$
a_{1} \cdot \mathbf{a}_{1}^{\mathbf{e m}} \oplus \cdots \oplus a_{|\mathbb{M}|} \cdot \mathbf{a}_{|\mathbb{M}|}^{\mathbf{e m}}=\mathbf{0} .
$$

For (19) to hold, the componentwise XOR of the columns of $\mathbf{A}^{\text {em }}$ that are associated with nonzero $a_{i}$ 's must be a zero column vector. In other words, each row of the matrix formed by the columns of $\mathbf{A}^{\mathbf{e m}}$ that are associated with nonzero $a_{i}$ 's must contain even number of 1 's. Therefore, $\mathbf{A}^{\mathbf{e m}}$ has a submatrix consisting of its columns which does not contain a row of weight one, indicating $\mathbb{M}$ is a dead-end set.

To show that the converse is incorrect, we display a counterexample. Consider $\mathbf{A}(15,3,3)$ with $q_{1}=3, q_{2}=4$ and $q_{3}=5$. It can be verified that $\{1,2,5,7,10,11\}$ is a dead-end set, but not a rank-deficient set.

The following result follows immediately from Theorem 9.

Corollary 1: For any group generation matrix $\mathbf{A}$, it holds that

$$
\begin{aligned}
N^{g}(\mathbf{A}) & \geq N^{e}(\mathbf{A}), \\
E^{g}(\mathbf{A}, i) & \leq E^{e}(\mathbf{A}, i) .
\end{aligned}
$$

\section{Performance Evaluation}

In this section, we present theoretical and simulated performance of the proposed scheme and compare it with that of the LDPC- and SSF-based ones [9]-[12].

\section{A. Study Setup}

Unless mentioned otherwise, we use the following setup.

- For comparison with [9]-[12], the size of the target collection of RFID tags is assumed to be equal to $n=25$, 49,121 or 169 , and, because aiming at minimizing the memory consumption of each RFID tag, group generation matrices with $j=2,3$ or 4 are considered. 
- Comparisons are conducted under the constraint of fixed $n$ and $j$, with which different group generation matrices have the same number of 1 's.

- We consider two performance metrics: error rate and total memory consumption. Because, to the best of our knowledge, there is no known efficient algorithm for finding rank-deficient or dead-end sets of any size [23], we have difficulty providing theoretical results of error rates for any given parameters. Therefore, we present theoretical error rates only for the small target collection size $n=25$ and mainly use simulation to evaluate different schemes.

- All simulation results presented below are mean values of $2 \cdot 10^{7}$ simulation runs. In each simulation run, missing RFID tags are selected uniformly at random from the target collection.

- Unique IDs have a length of 96 bits and are generated randomly and uniformly. In grouping, the hashes of unique IDs are calculated using CRC-32 and, therefore, each group ID is 32 bits in length, which is shorter than that of unique IDs. For simplicity, the memory consumptions of a group ID in grouping and its extended counterpart are normalized and assumed to be equal to $c_{1}=\frac{32}{96}=0.33$ and $c_{2}=1$, respectively, while that of a short ID is assumed to be equal to $c_{3}=\frac{8}{96}=0.083$.

- To distinguish the CRT-based scheme from the LDPCbased one, we use $[n, j, v]$ to denote the parameters of the CRT-based scheme.

- In the CRT-based scheme, the policy for selecting the pairwise relatively prime integers $q_{i}$ 's is based on the minimization of the number of groups.

\section{B. Using $v=2$}

In this subsection, we evaluate the performance of the CRTbased scheme under the scenario where $v=2$. Fig. 1 depicts the error rates of the LDPC-, SSF- and CRT-based grouping schemes with different values of $n$. Fig. 1 shows that the CRTbased scheme has lower error rates than the LDPC- and SSFbased ones. Fig. 1 also shows that the difference among the schemes vanishes when $n=121$ or 169 , and $j=4$. This is due to the use of CRC-32 as the HF in the simulation, which can increase linear dependence of the hashes of RFID tags compared to using the trivial HF. Fig. 1(a) also demonstrates that simulation results are well in accord with theoretical ones. Table I depicts the maximum number of missing RFID tags that can be determined within a specified tolerance of error rate. Table I shows that the maximum number of missing RFID tags that can be determined by the CRT-based scheme is higher than that provided by the LDPC- or SSF-based one. Table I also shows that, given fixed $n$ and $v$, the maximum number of missing RFID tags that can be determined increases with increasing $j$. This is mainly because a larger value of $j$ leads to a larger number of groups. In addition, Table I shows that the decoding guarantee of the CRT-based scheme is greater than that determined by Theorem 1 .

Fig. 2 depicts the error rates of the LDPC-, SSF- and CRTbased extended grouping schemes. Similar to grouping, Fig. 2 shows that the CRT-based scheme performs better than the LDPC- and SSF-based ones, especially for $j=4$. Fig. 2(a) also shows that simulation results agree with theoretical ones. Table II depicts the maximum number of missing RFID tags that can be identified within a specified tolerance of error rate. Table II shows that the maximum number of missing RFID tags that can be identified by the CRT-based scheme is higher than that provided by the LDPC- or SSF-based one. It also shows that the decoding guarantee of the CRT-based scheme is greater than that determined by Theorem 1 .

TABLE I

MAXIMUM NUMBER OF MISSING RFID TAGS THAT CAN BE DETERMINED WITHIN A SPECIFIED TOLERANCE OF ERROR RATE

\begin{tabular}{c|c||c|c|c|c|c|c}
\hline \multicolumn{2}{c||}{ Scheme } & \multicolumn{2}{c|}{ LDPC-based } & \multicolumn{2}{c|}{ SSF-based } & \multicolumn{2}{c}{ CRT-based } \\
\hline \multicolumn{2}{c||}{ Error rate } & $0 \%$ & $5 \%$ & $0 \%$ & $5 \%$ & $0 \%$ & $5 \%$ \\
\hline \hline \multirow{6}{*}{$(n, j)$} & $(25,2)$ & 1 & 2 & 3 & 5 & 3 & 5 \\
\cline { 2 - 8 } & $(25,3)$ & 3 & 8 & 5 & 9 & 5 & 12 \\
\cline { 2 - 8 } & $(25,4)$ & 5 & 13 & 7 & 13 & 10 & 16 \\
\cline { 2 - 8 } & $(49,2)$ & 1 & 3 & 3 & 6 & 3 & 6 \\
\cline { 2 - 8 } & $(49,3)$ & 1 & 9 & 5 & 13 & 5 & 16 \\
\cline { 2 - 8 } & $(49,4)$ & 3 & 19 & 7 & 19 & 8 & 25 \\
\cline { 2 - 8 } & $(121,2)$ & 1 & 4 & 3 & 9 & 3 & 9 \\
\cline { 2 - 8 } & $(121,3)$ & 1 & 13 & 5 & 21 & 5 & 24 \\
\hline & $(121,4)$ & 6 & 27 & 6 & 27 & 6 & 27 \\
\cline { 2 - 8 } & $(169,2)$ & 1 & 5 & 3 & 10 & 3 & 10 \\
\cline { 2 - 8 } & $(169,3)$ & 1 & 13 & 5 & 25 & 5 & 26 \\
\cline { 2 - 8 } & $(169,4)$ & 1 & 26 & 7 & 27 & 7 & 27 \\
\hline
\end{tabular}

TABLE II

MAXIMUM NUMBER OF MISSING RFID TAGS THAT CAN BE IDENTIFIED WITHIN A SPECIFIED TOLERANCE OF ERROR RATE

\begin{tabular}{|c|c|c|c|c|c|c|c|}
\hline \multirow{2}{*}{\multicolumn{2}{|c|}{$\begin{array}{c}\text { Scheme } \\
\text { Error rate }\end{array}$}} & \multicolumn{2}{|c|}{ LDPC-based } & \multicolumn{2}{|c|}{ SSF-based } & \multicolumn{2}{|c|}{ CRT-based } \\
\hline & & $0 \%$ & $5 \%$ & $0 \%$ & $5 \%$ & $0 \%$ & $5 \%$ \\
\hline \multirow{12}{*}{$(n, j)$} & $(25,2)$ & $\overline{1}$ & $\overline{2}$ & $\overline{3}$ & 5 & $\overline{3}$ & 5 \\
\hline & $(25,3)$ & 3 & 8 & 5 & 9 & 5 & 11 \\
\hline & $(25,4)$ & 5 & 11 & 7 & 11 & 9 & 15 \\
\hline & $(49,2)$ & 1 & 3 & 3 & 6 & 3 & 6 \\
\hline & $(49,3)$ & 1 & 9 & 5 & 12 & 5 & 15 \\
\hline & $(49,4)$ & 3 & 16 & 7 & 16 & 8 & 21 \\
\hline & $(121,2)$ & $\overline{1}$ & $\overline{4}$ & 3 & $\overline{9}$ & 3 & 9 \\
\hline & $(121,3)$ & 1 & 13 & 5 & 20 & 5 & 23 \\
\hline & $(121,4)$ & 6 & 27 & 13 & 27 & 15 & 33 \\
\hline & $(169,2)$ & 1 & 5 & 3 & $\overline{10}$ & 3 & 10 \\
\hline & $(169,3)$ & 1 & 13 & 5 & 24 & 5 & 27 \\
\hline & $(169,4)$ & 1 & 31 & 9 & 32 & 16 & 37 \\
\hline
\end{tabular}

By comparing Table I with Table II, one can see that, for a given group generation matrix, the decoding guarantee of grouping may be smaller than that of its extended counterpart. In addition, comparing Fig. 1 with Fig. 2 reveals that the error rate of grouping based on a group generation matrix may be larger than that of its extended counterpart. These observations are in stark contrast to Corollary 1. The reason for this is that, in the simulation, grouping uses CRC-32 as the HF, which can increase linear dependence of the hashes of RFID tags.

To investigate the effect of the choice of the pairwise relatively prime integers $q_{i}$ 's on the performance of the CRTbased scheme, Fig. 3 depicts the error rates of the CRT-based extended grouping scheme with $[25,2,2]$ where different pairs of $\left(q_{1}, q_{2}\right)$ 's are employed. More specifically, $q_{1}$ and $q_{2}$ are selected as two consecutive positive integers. For comparison, 


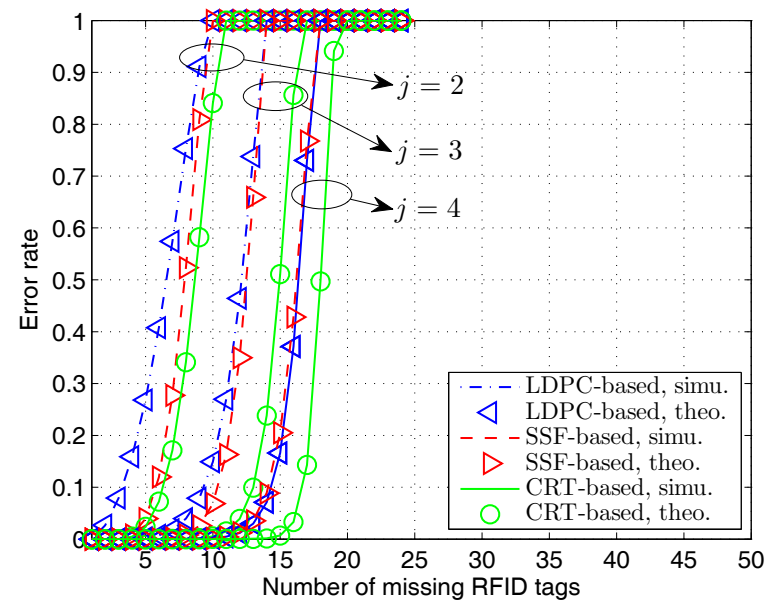

(a)

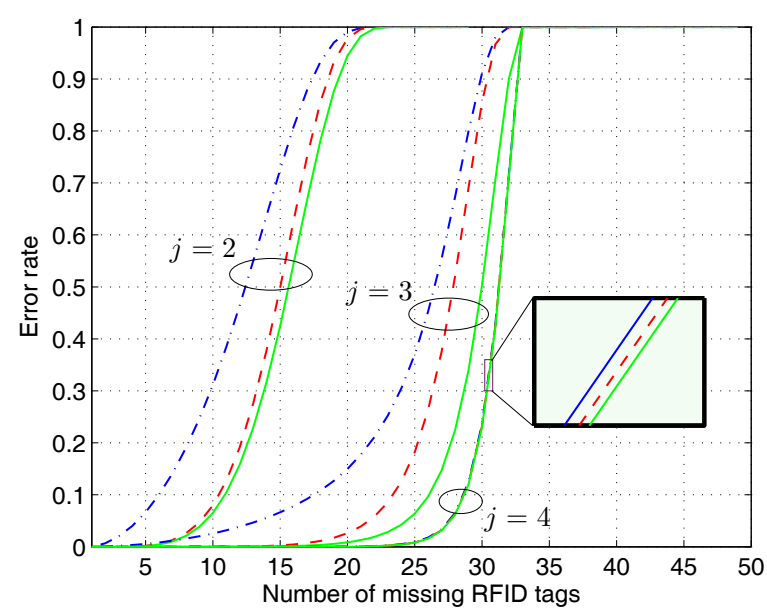

(c)

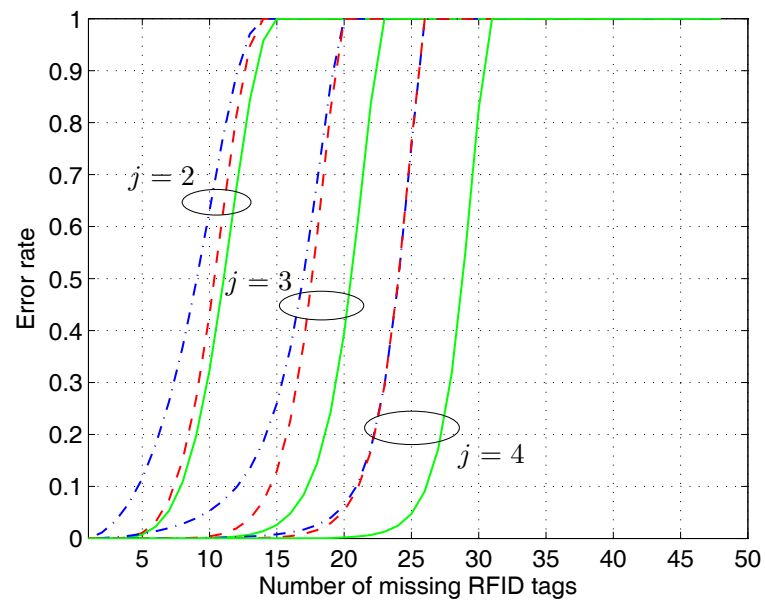

(b)

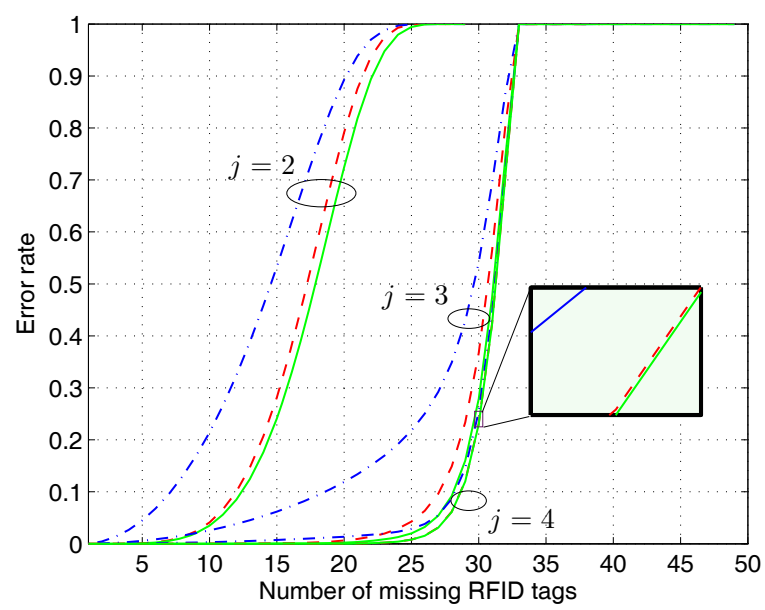

(d)

Fig. 1. Error rates of different grouping schemes: (a) $n=25$; (b) $n=49$; (c) $n=121$; (d) $n=169$.

the performance of the SSF-based extended grouping scheme is also included and labeled as the pair $(5,5)$, as each of the two submatrices in the group generation matrix has five rows. Fig. 3 shows that the pair $(8,9)$ has the lowest error rate. Table III depicts the decoding guarantees and total memory consumptions for different pairs of $\left(q_{1}, q_{2}\right)$ 's. Table III shows that the decoding guarantee of the SSF-based scheme is equal to three and that the decoding guarantee of the CRT-based scheme agrees with Theorem 6 . Although the pair $(8,9)$ can only provide the same decoding guarantee as the SSF-based scheme, its error rate is much better, indicating that a larger number of groups helps in reducing error rates. Table III also shows that the total memory consumption decreases as the values of $q_{1}$ and $q_{2}$ increase. This is because each group contains fewer RFID tags with increasing $q_{1}$ and $q_{2}$, which reduces memory consumptions of storing short IDs. Therefore, for the CRT-based extended grouping scheme, larger values of $q_{i}$ 's not only can lower error rates, but also minimizes total memory consumptions.

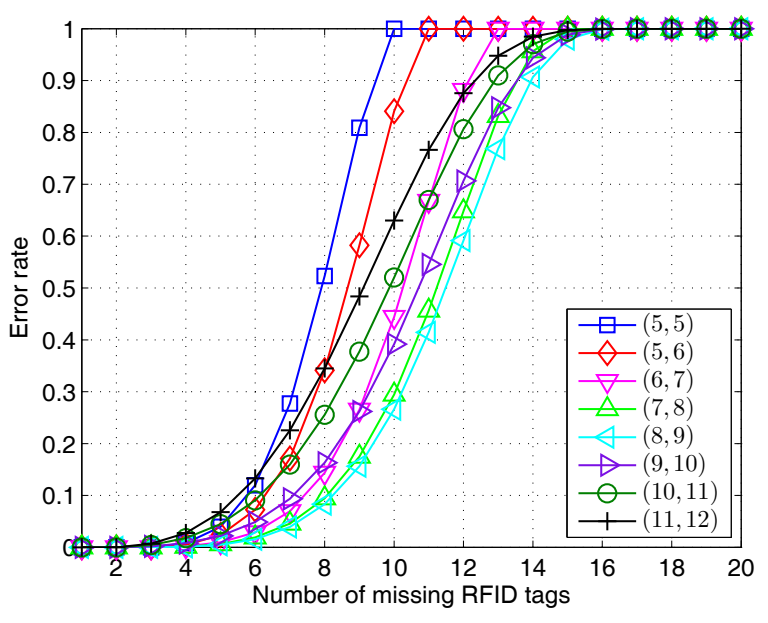

Fig. 3. Error rates of different extended grouping schemes with different pairs of $\left(q_{1}, q_{2}\right)$ 's. 


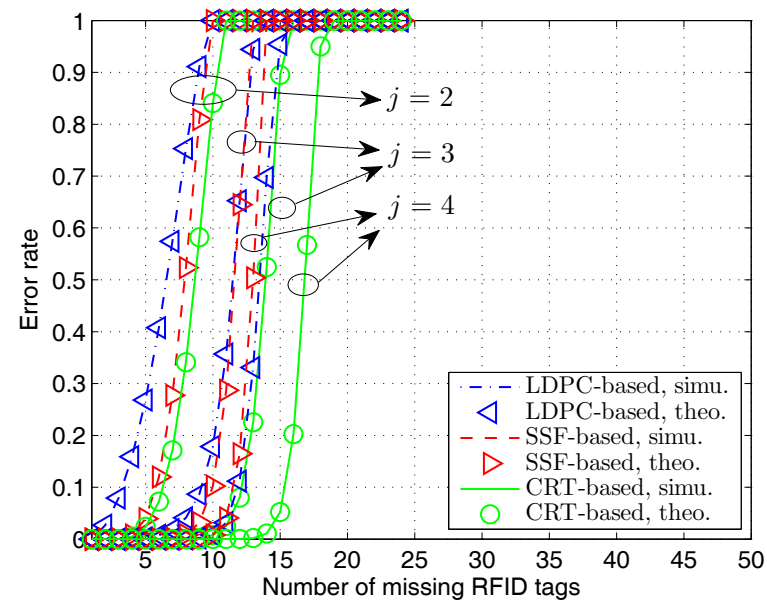

(a)

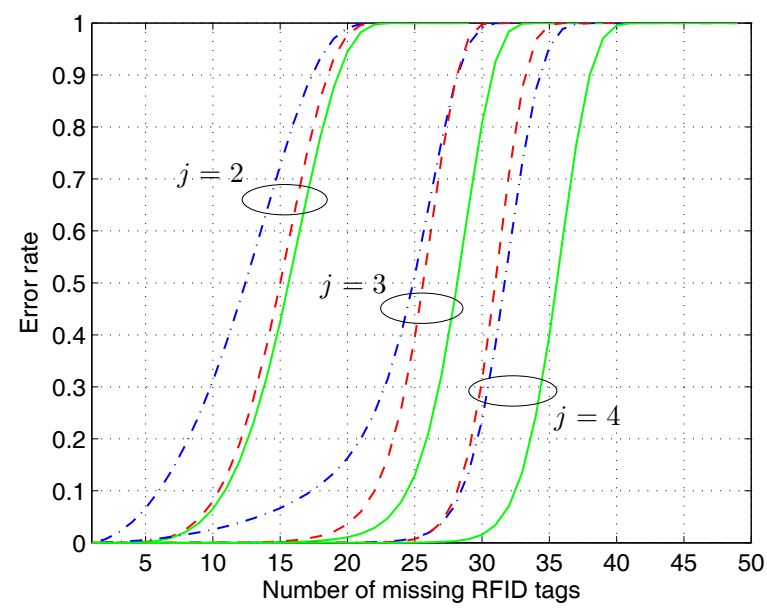

(c)

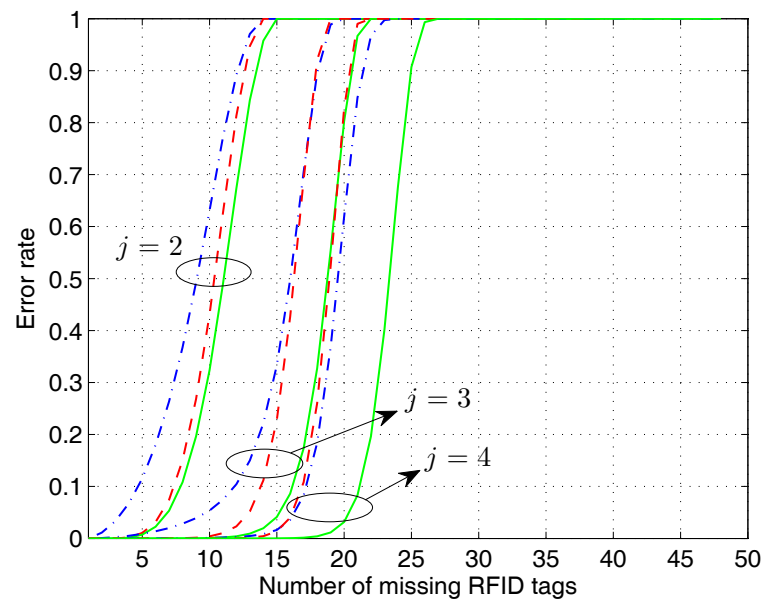

(b)

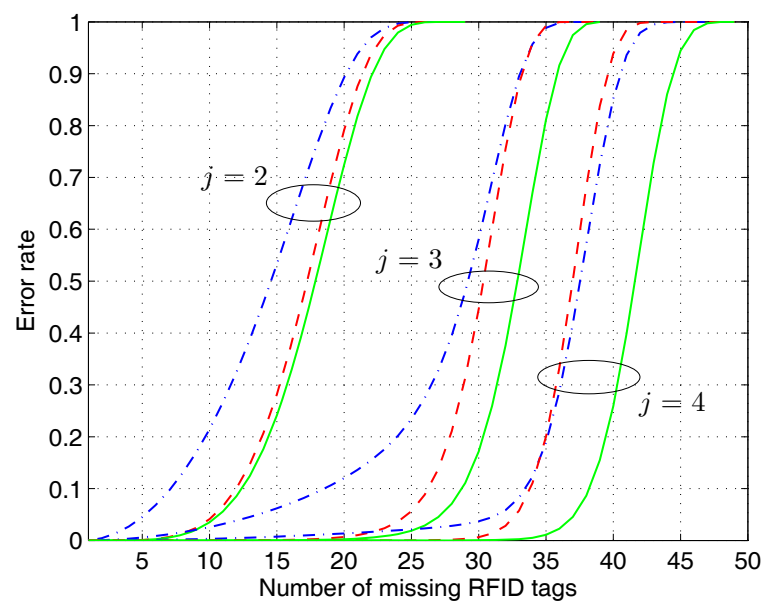

(d)

Fig. 2. Error rates of different extended grouping schemes: (a) $n=25$; (b) $n=49$; (c) $n=121$; (d) $n=169$.

TABLE III

DECODING GUARANTEES $N^{e}$ 'S AND TOTAL MEMORY CONSUMPTIONS $M^{e}$ 'S OF DIFFERENT EXTENDED GROUPING SCHEMES WITH DIFFERENT PAIRS OF $\left(q_{1}, q_{2}\right)$ 's

\begin{tabular}{l|l||c|c}
\hline Scheme & $\left(q_{1}, q_{2}\right)$ & $N^{e}$ & $M^{e}$ \\
\hline \hline SSF-based & $(5,5)$ & 3 & 66.67 \\
\hline \multirow{5}{*}{ CRT-based } & $(5,6)$ & 3 & 65.00 \\
\cline { 2 - 4 } & $(6,7)$ & 3 & 62.17 \\
\cline { 2 - 4 } & $(7,8)$ & 3 & 60.00 \\
\cline { 2 - 4 } & $(8,9)$ & 3 & 58.33 \\
\cline { 2 - 4 } & $(9,10)$ & 2 & 57.17 \\
\cline { 2 - 4 } & $(10,11)$ & 2 & 56.17 \\
\cline { 2 - 4 } & $(11,12)$ & 2 & 55.17 \\
\hline
\end{tabular}

\section{Using $v=3$}

In this subsection, we investigate the effect of using $v=3$ on the performance of the proposed scheme. For demonstration, we focus our attention mainly on the CRT-based grouping scheme. Fig. 4 depicts the error rates of the CRTbased grouping scheme with different $[25, j, v]$ 's. In addition to simulation results using CRC-32 as the HF, Fig. 4 depicts simulation results using CRC-16, in which each group ID is 16 bits in length. To demonstrate tradeoffs between $j$ and $v$ that are shown in Fig. 4, Table IV depicts the pairwise relatively prime integers $q_{i}$ 's, decoding guarantees and total memory consumptions of the CRT-based grouping scheme with different $[25, j, v]$ 's. Fig. 4 shows that simulation results using CRC-32 are well in accord with theoretical ones and that simulation results using CRC-16 fails to match theoretical ones except when $[25,3,3]$ is used. Fig. 4 also shows that, given fixed $n=25$ and $j=3$, increasing $v$ from two to three increases the error rate, although it allows to reduce the number of groups from 18 to 10 such that the assumption of linear independence of the hashes of RFID tags can more easily hold. The increase in error rates can be explained easily through Theorem 1: the decoding guarantee determined by Theorem 1, i.e., $1+\left\lfloor\frac{j-1}{v-1}\right\rfloor$, decreases from three to two, as depicted in Table IV. To use $v=3$ while maintaining the same decoding guarantee determined by Theorem 1, we have to increase $j$ to five, by which the error rate improves, as shown in Fig. 4. The total memory consumption, however, increases 


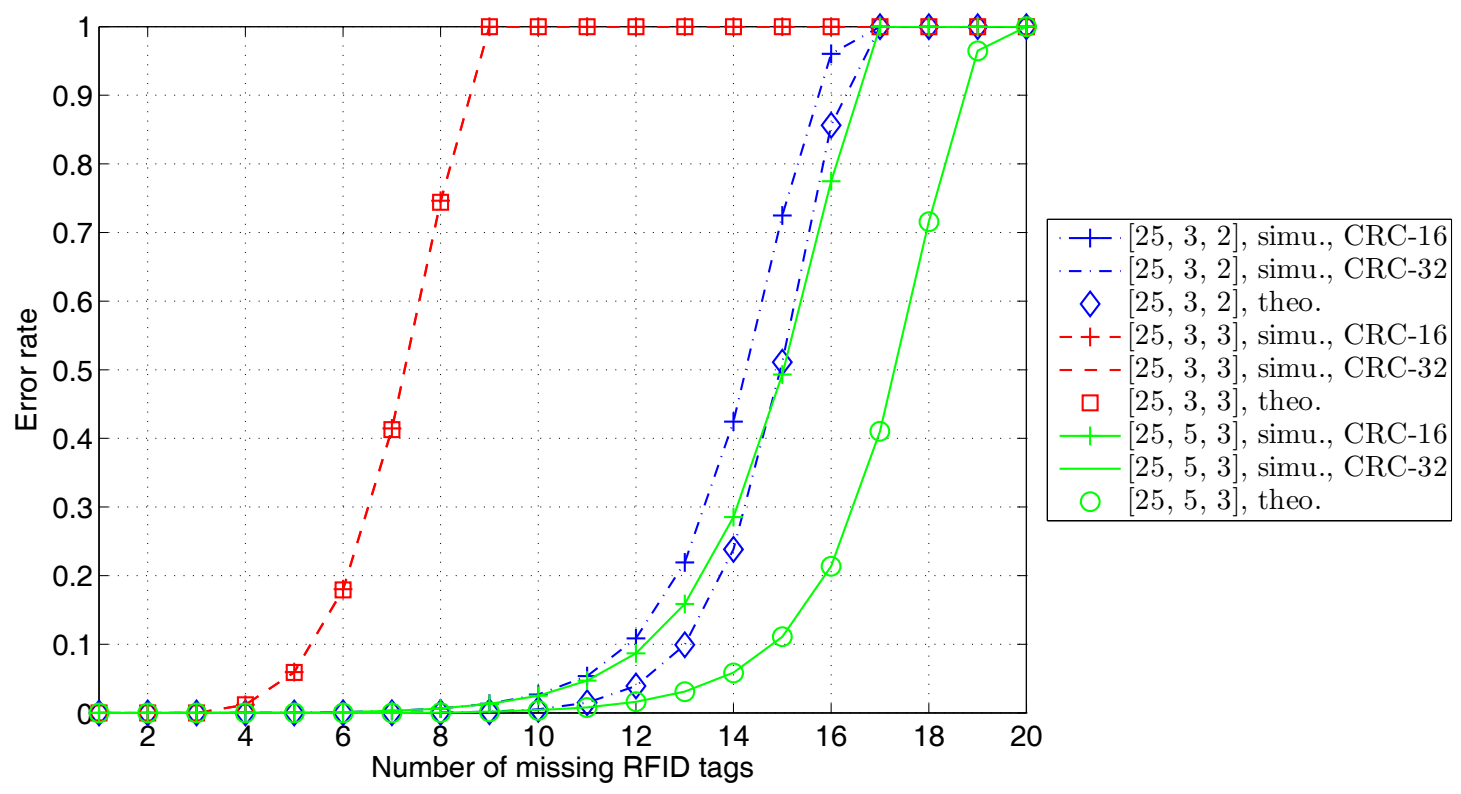

Fig. 4. Error rates of the CRT-based grouping scheme with different $[25, j, v]$ 's.

accordingly, as shown in Table IV. Table IV also demonstrates another advantage of using a larger value of $v$ : less total memory consumption. This is because the policy for selecting $q_{i}$ 's is based on the minimization of the number of groups and because HFs producing shorter hashes are sufficient for the assumption of linear independence of the hashes of RFID tags to hold.

TABLE IV

PAIRWISE RELATIVELY PRIME INTEGERS $q_{i}$ 'S, DECODING GUARANTEES $N^{g}$ 'S AND TOTAL MEMORY CONSUMPTIONS $M^{g}$ 'S OF THE CRT-BASED GROUPING SCHEME WITH DIFFERENT $[25, j, v]$ 's

\begin{tabular}{|c|c|c|c|c|c|c|}
\hline \multicolumn{2}{|c|}{$[n, j, v]$} & $q_{i}$ 's & $1+$ & $\frac{j-1}{v-1}$ & $N^{g}$ & $M^{g}$ \\
\hline \multirow{3}{*}[25,3,2]{} & 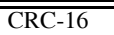 & \multirow{3}{*}{$5,6,7$} & \multirow{3}{*}{\multicolumn{2}{|c|}{3}} & 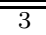 & $=12.50$ \\
\hline & CRC-32 & & & & 5 & 25.00 \\
\hline & Trivial HF & & & & 5 & 75.00 \\
\hline \multirow{3}{*}[25,3,3]{} & CRC-16 & \multirow{3}{*}{$2,3,5$} & \multirow{3}{*}{\multicolumn{2}{|c|}{2}} & 3 & 12.50 \\
\hline & CRC-32 & & & & 3 & 25.00 \\
\hline & Trivial HF & & & & 3 & 75.00 \\
\hline \multirow{3}{*}[25,5,3]{} & CRC-16 & \multirow{3}{*}{$2,3,5,7,11$} & \multirow{3}{*}{\multicolumn{2}{|c|}{3}} & 3 & 20.83 \\
\hline & CRC-32 & & & & 5 & 41.67 \\
\hline & Trivial HF & & & & 5 & 125.00 \\
\hline
\end{tabular}

\section{CONCLUSION}

In this paper, we has proposed a Chinese-remaindertheorem-based scheme for the design of grouping of RFID tags. The proposed scheme not only provides decoding guarantees, but also offers flexibility in constructing group generation matrices. The proposed scheme has proved to be an efficient approach to the design of grouping of RFID tags. Also, we have characterized the key objects needed to study decoding guarantees of grouping and its extended counterpart, called rank-deficient and dead-end sets, respectively, which enable theoretical analyses of error rates. The two key objects are related to the minimum and stopping distances of a linear code, respectively. As such, the characterization has offered direct connection with coding theory that helps in the understanding of the verification/identification problems being studied.

\section{REFERENCES}

[1] M. Bolic, D. Simplot-Ryl, and I. Stojmenovic, RFID Systems: Research Trends and Challenges, New York: Wiley, 2010.

[2] D. K. Klair, K.-W. Chin, and R. Raad, "A survey and tutorial of RFID anti-collision protocols," IEEE Commun. Surveys and Tutorials, vol. 12, pp. 400-421, 2010.

[3] M. Pargett, What is EDI? A Guide to Electronic Data Interchange, 2nd ed. Oxford, UK: NCC Blackwell, 1992.

[4] A. Juels, "Yoking-proofs for RFID tags," in Proc. 2nd IEEE Annu. Conf Pervasive Comput. Commun. Workshops, 2004, pp. 138-143.

[5] J. Saito and K. Sakurai, "Grouping proof for RFID tags," in Proc. 19th Conf. Adv. Inform. Netw. Appl. (AINA), 2005, pp. 621-624.

[6] L. Bolotnyy and G. Robins, "Generalized yoking-proofs for a group of RFID tags," in Proc. Int. Conf. Mobile and Ubiquitous Syst. (Mobiquitous), 2006, pp. 1-4.

[7] S. Inoue, D. Hagiwara, and H. Yasuura, "Systematic error detection for RFID reliability," in Proc. 1st Int. Conf. Availability, Reliability and Security (ARES), 2006,

[8] V. Potdar, P. Hayati, and E. Chang, "Improving RFID read rate reliability by a systematic error detection approach," in Proc. 1st Annu. RFID Eurasia, 2007, pp. 1-5.

[9] Y. Sato, J. Mitsugi, O. Nakamura, and J. Murai, "Group coding of RF tags to verify the integrity of group of objects," in Proc. IEEE RFID, Apr. 2011, pp. 200-206.

[10] Y. Sato, J. Mitsugi, O. Nakamura, and J. Murai, "Theory and performance evaluation of group coding of RFID tags," IEEE Trans. Autom. Sci. Eng., vol. 9, pp. 458-466, July 2012.

[11] Y. Sato, Y. Igarashi, J. Mitsugi, O. Nakamura, and J. Murai, "Identification of missing objects with group coding of RF tags," in Proc. IEEE RFID, Apr. 2012, pp. 95-101.

[12] Y.-S. Su, J.-R. Lin, and O. K. Tonguz, "Grouping of RFID tags via strongly selective families," IEEE Commun. Lett., vol. 17, pp. 11201123 , June 2013.

[13] R. Gallager, "Low-density parity-check codes," IRE Trans. Inf. Theory, vol. 8, pp. 21-28, Jan. 1962.

[14] A. Clementi, A. Monti, and R. Silvestri, "Distributed broadcast in radio networks of unknown topology," Theoret. Comput. Sci., vol. 302, pp. 337-364, 2003. 
[15] C. Ding, D. Pei, and A. Salomaa, Chinese Remainder TheoremApplications in Computing, Coding, Cryptography, Singapore: World Scientific, 1996.

[16] O. Goldreich, D. Ron, and M. Sudan, "Chinese remaindering with errors," IEEE Trans. Inf. Theory, vol. 46, pp. 1330-1338, July 2000.

[17] W. Kautz and R. Singleton, "Nonrandom binary superimposed codes," IEEE Trans. Inf. Theory, vol. 10, pp. 363-377, Oct. 1964.

[18] P. Erdös, P. Frankl, and Z. Füredi, "Families of finite sets in which no set is covered by the union of $r$ others," Israel J. Math., vol. 51, pp. 79-89, 1985.

[19] D.-Z. Du and F. Hwang, Combinatorial Group Testing and Its Applications, 2nd ed. Singapore: World Scientific, 2000.

[20] S. B. Wicker, Error Control Systems for Digital Communication and Storage, Prentice-Hall, 1995.

[21] J. H. Weber and K. A. S. Abdel-Ghaffar, "Results on parity-check matrices with optimal stopping and/or dead-end set enumerators," IEEE Trans. Inf. Theory, vol. 54, pp. 1368-1374, Mar. 2008.

[22] C. Di, D. Proietti, I. E. Telatar, T. J. Richardson, and R. L. Urbanke, "Finite-length analysis of low-density parity-check codes on the binary erasure channel," IEEE Trans. Inf. Theory, vol. 48, pp. 1570-1579, June 2002.

[23] K. M. Krishnan and P. Shankar, "Computing the stopping distance of a Tanner graph is NP-hard," IEEE Trans. Inf. Theory, vol. 53, pp. 22782280, June 2007.

[24] M. Esmaeili and M. J. Amoshahy, "On the stopping distance of array code parity-check matrices," IEEE Trans. Inf. Theory, vol. 55, pp. 3488 3493, Aug. 2009.

[25] M. Esmaeili, M. H. Tadayon, and T. A. Gulliver, "More on the stopping and minimum distances of array codes," IEEE Trans. Commun., vol. 59, pp. 750-757, Mar. 2011. 